\title{
Isotropic Immersions of Complex Space Forms into Real Space Forms and Mean Curvatures
}

by

\author{
Nobutaka BOUMUKI
}

Presented by Bogdan BOJARSKI

Summary. Using an inequality related to the mean curvature, we give a sufficient condition for an isotropic immersion of a complex space form into a real space form to be parallel.

1. Introduction. Parallel submanifolds of real space forms are ones of the most interesting objects in differential geometry (cf. $[\mathrm{F}-1, \mathrm{~T}]$ ). Here, we have in mind the following fact: "all parallel immersions of Riemannian symmetric spaces of rank one into a real space form are isotropic but there exist many isotropic immersions of these spaces into a real space form, which are not parallel." For example, the fourth standard minimal immersion $f$ : $S^{3}(1 / 8) \rightarrow S^{24}(1)$ is isotropic, but not parallel. So, sufficient conditions for these isotropic immersions to be parallel are worth considering. In this paper, we are interested in parallel immersions of a complex space form into a real space form.

We have proved the following theorem in the previous paper [B]:

Theorem A. Let $f$ be a $\lambda$-isotropic immersion of an $n(\geq 2)$-dimensional compact oriented real space form $M^{n}(c ; \mathbb{R})$ of constant sectional curvature $c$ into an m-dimensional real space form $\widetilde{M}^{m}(\tilde{c} ; \mathbb{R})$ of constant sectional curvature $\tilde{c}$. Suppose that the mean curvature vector field $\mathfrak{h}$ and the mean curvature $H=\|\mathfrak{h}\|$ satisfy the following two inequalities:

(i) $H^{2} \leq \frac{2(n+1)}{n} c-\tilde{c}$,

(ii) $0 \leq(1-n) \Delta H^{2}+n\langle\mathfrak{h}, \Delta \mathfrak{h}\rangle$,

2000 Mathematics Subject Classification: 53B25, 53C40.

Key words and phrases: isotropic immersions, parallel immersions, real space forms, complex space forms, quaternionic space forms. 
where $\Delta$ denotes the Laplacian on $M^{n}(c ; \mathbb{R})$. Then $f$ is a parallel immersion. Moreover $f$ is locally equivalent to one of the following:

(I) $f$ is a totally umbilic immersion of $S^{n}(c)$ into $\widetilde{M}^{m}(\tilde{c} ; \mathbb{R})$, where $c \geq \tilde{c}$ and $H^{2} \equiv c-\tilde{c}$.

(II) $f=f_{2} \circ f_{1}: S^{n}(c) \stackrel{f_{1}}{\longrightarrow} S^{n+n(n+1) / 2-1}(2(n+1) c / n) \stackrel{f_{2}}{\longrightarrow} \widetilde{M}^{m}(\tilde{c} ; \mathbb{R})$, where $f_{1}$ is the second standard minimal immersion, $f_{2}$ is a totally umbilic immersion, $2(n+1) c / n \geq \tilde{c}$ and $H^{2} \equiv 2(n+1) c / n-\tilde{c}$.

REMARK 1. We remark that Theorem $\mathrm{A}$ is no longer true if we omit condition (ii).

In this paper, we consider the case where the submanifold is a complex space form, and get the following theorem:

TheOREM 1. Let $f$ be a $\lambda$-isotropic immersion of a complex $n(\geq 2)$ dimensional complex space form $M^{n}(4 c ; \mathbb{C})$ of constant holomorphic sectional curvature $4 c$ into an $m$-dimensional real space form $\widetilde{M}^{m}(\tilde{c} ; \mathbb{R})$ of constant sectional curvature $\tilde{c}$. Suppose that the mean curvature $H$ satisfies

$$
H^{2} \leq \frac{2(n+1)}{n} c-\tilde{c} .
$$

Then $f$ is a parallel immersion. Moreover $f$ is locally equivalent to one of the following:

(I) $f$ is a totally geodesic immersion of $\mathbb{C}^{n}\left(=\mathbb{R}^{2 n}\right)$ into $\mathbb{R}^{m}$, where $H \equiv 0$.

(II) $f$ is a totally umbilic immersion of $\mathbb{C}^{n}$ into $\mathbb{R} H^{m}(\tilde{c})$, where $H^{2} \equiv$ $-\tilde{c}$.

(III) $f=f_{2} \circ f_{1}: \mathbb{C} P^{n}(4 c) \stackrel{f_{1}}{\longrightarrow} S^{n^{2}+2 n-1}(2(n+1) c / n) \stackrel{f_{2}}{\longrightarrow} \widetilde{M^{m}}(\tilde{c} ; \mathbb{R})$, where $f_{1}$ is the first standard minimal immersion, $f_{2}$ is a totally umbilic immersion, $2(n+1) c / n \geq \tilde{c}$ and $H^{2} \equiv 2(n+1) c / n-\tilde{c}$.

The main purpose of this paper is to prove Theorem 1 .

REMARK 2. It is interesting to compare Theorem A with Theorem 1: the submanifold in Theorem 1 need not be compact, and Theorem 1 does not require condition (ii) of Theorem $\mathrm{A}$.

The author is grateful to Professor Sadahiro Maeda for his valuable suggestions, and thanks the referee for his good advice.

2. Preliminaries. Let $f: M \rightarrow \widetilde{M}$ be an isometric immersion of a Riemannian manifold $M$ into a Riemannian manifold $\widetilde{M}$ with metric $\langle$, $\rangle$, and $\sigma$ the second fundamental form. We recall the notion of isotropic immersion (cf. [O]): the immersion $f$ is said to be isotropic if $\|\sigma(X, X)\| /\|X\|^{2}$ is constant for all $x \in M$ and all tangent vectors $X(\neq 0)$ to $M$ at $x$. If we define 
the function $\lambda$ on $M$ by $x(\in M) \mapsto\|\sigma(X, X)\| /\|X\|^{2}$, then the immersion $f$ is also said to be $\lambda$-isotropic. Note that totally umbilic immersions are isotropic, but not vice versa.

An $n$-dimensional real space form $M^{n}(c ; \mathbb{R})$ is a Riemannian manifold of constant sectional curvature $c$, which is locally congruent to a standard sphere $S^{n}(c)$, a Euclidean space $\mathbb{R}^{n}$ or a real hyperbolic space $\mathbb{R} H^{n}(c)$, according as $c$ is positive, zero or negative. An $n$-dimensional complex space form $M^{n}(c ; \mathbb{C})$ is a Kähler manifold of constant holomorphic sectional curvature $c$, which is locally congruent to a complex projective space $\mathbb{C} P^{n}(c)$, a complex Euclidean space $\mathbb{C}^{n}\left(=\mathbb{R}^{2 n}\right)$ or a complex hyperbolic space $\mathbb{C} H^{n}(c)$, according as $c$ is positive, zero or negative. An $n$-dimensional quaternionic space form $M^{n}(c ; \mathbb{Q})$ is a quaternionic Kähler manifold of constant quaternionic sectional curvature $c$, which is locally congruent to a quaternionic projective space $\mathbb{Q} P^{n}(c)$, a quaternionic Euclidean space $\mathbb{Q}^{n}$ $\left(=\mathbb{R}^{4 n}\right)$ or a quaternionic hyperbolic space $\mathbb{Q} H^{n}(c)$, according as $c$ is positive, zero or negative.

3. Proof of Theorem 1. Let $J$ be the complex structure on $M^{n}(4 c ; \mathbb{C})$. Then the curvature tensor $R$ of $M^{n}(4 c ; \mathbb{C})$ is given by

$$
\begin{array}{rl}
R(X, Y) Z=c & c\langle\langle Y, Z\rangle X-\langle X, Z\rangle Y+\langle J Y, Z\rangle J X \\
& -\langle J X, Z\rangle J Y+2\langle X, J Y\rangle J Z\}
\end{array}
$$

for all $X, Y, Z \in \mathfrak{X}\left(M^{n}(4 c ; \mathbb{C})\right)$, where we denote by $\mathfrak{X}\left(M^{n}(4 c ; \mathbb{C})\right)$ the set of all vector fields on $M^{n}(4 c ; \mathbb{C})$.

Since $f$ is $\lambda$-isotropic, we have $\langle\sigma(X, X), \sigma(X, X)\rangle=\lambda^{2}\langle X, X\rangle\langle X, X\rangle$ for all $X \in \mathfrak{X}\left(M^{n}(4 c ; \mathbb{C})\right)$, which is equivalent to

$$
\begin{array}{r}
\langle\sigma(X, Y), \sigma(Z, W)\rangle+\langle\sigma(X, Z), \sigma(W, Y)\rangle+\langle\sigma(X, W), \sigma(Y, Z)\rangle \\
=\lambda^{2}\{\langle X, Y\rangle\langle Z, W\rangle+\langle X, Z\rangle\langle W, Y\rangle+\langle X, W\rangle\langle Y, Z\rangle\}
\end{array}
$$

for all $X, Y, Z, W \in \mathfrak{X}\left(M^{n}(4 c ; \mathbb{C})\right)$.

The Gauss equation is written as follows:

$$
\begin{aligned}
& \langle\sigma(X, Y), \sigma(Z, W)\rangle-\langle\sigma(Z, Y), \sigma(X, W)\rangle \\
& =\langle R(Z, X) Y, W\rangle-\tilde{c}\{\langle X, Y\rangle\langle Z, W\rangle-\langle Z, Y\rangle\langle X, W\rangle\}
\end{aligned}
$$

for all $X, Y, Z, W \in \mathfrak{X}\left(M^{n}(4 c ; \mathbb{C})\right)$.

It follows from (3.1)-(3.3) that

$$
\begin{aligned}
\langle\sigma(X, Y), \sigma(Z, W)\rangle= & \frac{\lambda^{2}+2(c-\tilde{c})}{3}\langle X, Y\rangle\langle Z, W\rangle \\
& +\frac{\lambda^{2}-(c-\tilde{c})}{3}\{\langle X, W\rangle\langle Y, Z\rangle+\langle X, Z\rangle\langle Y, W\rangle\} \\
& +c\{\langle J X, W\rangle\langle J Y, Z\rangle+\langle J X, Z\rangle\langle J Y, W\rangle\}
\end{aligned}
$$


for all $X, Y, Z, W \in \mathfrak{X}\left(M^{n}(4 c ; \mathbb{C})\right)$. This yields

$$
\begin{gathered}
H^{2}=\frac{(n+1) \lambda^{2}+2(n+1) c-(2 n-1) \tilde{c}}{3 n}, \\
\|\sigma(X, J X)\|^{2}=\frac{\lambda^{2}-4 c+\tilde{c}}{3},
\end{gathered}
$$

where $X \in \mathfrak{X}\left(M^{n}(4 c ; \mathbb{C})\right)$ with $\|X\|=1$. It follows from (3.5) and (3.6) that

$$
H^{2}-\frac{2(n+1)}{n} c+\tilde{c}=\frac{n+1}{n}\|\sigma(X, J X)\|^{2} \geq 0,
$$

where $X \in \mathfrak{X}\left(M^{n}(4 c ; \mathbb{C})\right)$ with $\|X\|=1$.

Therefore, $H^{2} \equiv 2(n+1) c / n-\tilde{c}$ by assumption, so that

$$
\sigma(X, J X)=0
$$

for all $X \in \mathfrak{X}\left(M^{n}(4 c ; \mathbb{C})\right)$. From (3.7), we get

$$
\sigma(X, Y)=\sigma(J X, J Y)
$$

for all $X, Y \in \mathfrak{X}\left(M^{n}(4 c ; \mathbb{C})\right)$. Consequently, the immersion $f$ is parallel (cf. $[\mathrm{F}-2])$. The classification theorem for parallel submanifolds of a real space form completes the proof (cf. $[\mathrm{F}-1, \mathrm{~T}])$.

4. Quaternionic case. In this section, we consider the case where the submanifold is a quaternionic space form, and get the following theorem:

THEOREM 2. Let $f$ be a $\lambda$-isotropic immersion of a quaternionic $n(\geq 2)$ dimensional quaternionic space form $M^{n}(4 c ; \mathbb{Q})$ of constant quaternionic sectional curvature $4 c$ into an $m$-dimensional real space form $\widetilde{M}^{m}(\tilde{c} ; \mathbb{R})$ of constant sectional curvature $\tilde{c}$. Suppose that the mean curvature $H$ satisfies

$$
H^{2} \leq \frac{2(n+1)}{n} c-\tilde{c} .
$$

Then $f$ is a parallel immersion. Moreover $f$ is locally equivalent to one of the following:

(I) $f$ is a totally geodesic immersion of $\mathbb{Q}^{n}\left(=\mathbb{R}^{4 n}\right)$ into $\mathbb{R}^{m}$, where $H \equiv 0$.

(II) $f$ is a totally umbilic immersion of $\mathbb{Q}^{n}$ into $\mathbb{R} H^{m}(\tilde{c})$, where $H^{2} \equiv$ $-\tilde{c}$.

(III) $f=f_{2} \circ f_{1}: \mathbb{Q} P^{n}(4 c) \stackrel{f_{1}}{\longrightarrow} S^{2 n^{2}+3 n-1}(2(n+1) c / n) \stackrel{f_{2}}{\longrightarrow} \widetilde{M}^{m}(\tilde{c} ; \mathbb{R})$, where $f_{1}$ is the first standard minimal immersion, $f_{2}$ is a totally umbilic immersion, $2(n+1) c / n \geq \tilde{c}$ and $H^{2} \equiv 2(n+1) c / n-\tilde{c}$. 
Proof. Let $\{I, J, K\}$ be the canonical local basis on $M^{n}(4 c ; \mathbb{Q})$. Then the curvature tensor $R$ of $M^{n}(4 c ; \mathbb{Q})$ is given by

$$
\begin{aligned}
R(X, Y) Z= & c\{\langle Y, Z\rangle X-\langle X, Z\rangle Y+\langle I Y, Z\rangle I X-\langle I X, Z\rangle I Y \\
& +\langle J Y, Z\rangle J X-\langle J X, Z\rangle J Y+\langle K Y, Z\rangle K X \\
& -\langle K X, Z\rangle K Y+2\langle X, I Y\rangle I Z \\
& +2\langle X, J Y\rangle J Z+2\langle X, K Y\rangle K Z\}
\end{aligned}
$$

for all $X, Y, Z \in \mathfrak{X}\left(M^{n}(4 c ; \mathbb{Q})\right)$. It follows from (3.2), (3.3) and (4.1) that

$$
\begin{aligned}
\langle\sigma(X, Y), \sigma(Z, W)\rangle= & \frac{\lambda^{2}+2(c-\tilde{c})}{3}\langle X, Y\rangle\langle Z, W\rangle \\
& +\frac{\lambda^{2}-(c-\tilde{c})}{3}\{\langle X, W\rangle\langle Y, Z\rangle \\
& +\langle X, Z\rangle\langle Y, W\rangle\}+c\{\langle I X, W\rangle\langle I Y, Z\rangle \\
& +\langle I X, Z\rangle\langle I Y, W\rangle+\langle J X, W\rangle\langle J Y, Z\rangle \\
& +\langle J X, Z\rangle\langle J Y, W\rangle+\langle K X, W\rangle\langle K Y, Z\rangle \\
& +\langle K X, Z\rangle\langle K Y, W\rangle\}
\end{aligned}
$$

for all $X, Y, Z, W \in \mathfrak{X}\left(M^{n}(4 c ; \mathbb{Q})\right)$. Equation (4.2) yields

$$
\begin{aligned}
H^{2} & =\frac{(2 n+1) \lambda^{2}+4(n+2) c-(4 n-1) \tilde{c}}{6 n} \\
\|\sigma(X, I X)\|^{2} & =\|\sigma(X, J X)\|^{2}=\|\sigma(X, K X)\|^{2}=\frac{\lambda^{2}-4 c+\tilde{c}}{3},
\end{aligned}
$$

where $X \in \mathfrak{X}\left(M^{n}(4 c ; \mathbb{Q})\right)$ with $\|X\|=1$. It follows from (4.3) and (4.4) that

$$
\begin{aligned}
H^{2}-\frac{2(n+1)}{n} c+\tilde{c} & =\frac{2 n+1}{2 n}\|\sigma(X, I X)\|^{2}=\frac{2 n+1}{2 n}\|\sigma(X, J X)\|^{2} \\
& =\frac{2 n+1}{2 n}\|\sigma(X, K X)\|^{2} \geq 0,
\end{aligned}
$$

where $X \in \mathfrak{X}\left(M^{n}(4 c ; \mathbb{Q})\right)$ with $\|X\|=1$.

Therefore, $H^{2} \equiv 2(n+1) c / n-\tilde{c}$ by assumption, so that

$$
\sigma(X, I X)=\sigma(X, J X)=\sigma(X, K X)=0
$$

for all $X \in \mathfrak{X}\left(M^{n}(4 c ; \mathbb{Q})\right)$. From $(4.5)$, we get

$$
\sigma(X, Y)=\sigma(I X, I Y)=\sigma(J X, J Y)=\sigma(K X, K Y)
$$

for all $X, Y \in \mathfrak{X}\left(M^{n}(4 c ; \mathbb{Q})\right)$. Consequently, $f$ is parallel (cf. $\left.[\mathrm{M}]\right)$. The classification theorem for parallel submanifolds of a real space form completes the proof (cf. $[\mathrm{F}-1, \mathrm{~T}])$. 


\section{References}

[B] N. Boumuki, Isotropic immersions and parallel immersions of space forms into space forms, Tsukuba J. Math. 28 (2004), 117-126.

[F-1] D. Ferus, Immersions with parallel second fundamental form, Math. Z. 140 (1974), $87-92$.

[F-2] —, Symmetric submanifolds of Euclidean space, Math. Ann. 247 (1980), 81-93.

[M] S. Maeda, Remarks on isotropic immersions, Yokohama Math. J. 34 (1986), 83-90.

[O] B. O'Neill, Isotropic and Kähler immersions, Canad. J. Math. 17 (1965), 905-915.

[T] M. Takeuchi, Parallel submanifolds of space forms, in: Manifolds and Lie Groups, in honor of Yozô Matsushima, Birkhäuser, Boston, 1981, 429-447.

Nobutaka Boumuki

Department of Mathematics

Faculty of Science and Engineering

Shimane University

Matsue 690-8504, Japan

E-mail: boumuki@math.shimane-u.ac.jp

Received June 22, 2004;

received in final form November 8, 2004 